Discussion This case presented a significant diagnostic challenge to our team as the VVM possessed numerous idiosyncratic features prevalent in cases of dermoid cysts, with simultaneous findings that are contraindicative of most venous malformations. The regression of the VVM during the patient's pregnancy was counterintuitive to expectation, as endocrine fluctuations would be expected to precipitate rapid enlargement of the lesion. Similarly, the abnormal firmness of the mass discouraged the diagnosis of a VVM which is characteristically soft on palpation, but this may be explained by the presence of partially thrombosed vessels. We report the case and propose that catheter angiography remains important in cases where vascular malformation is considered.

Disclosures A. Dmytriw: None. J. Song: None. P. Gullane: None. K. terBrugge: None. E. Yu: None.

\section{E-083 ACUTE STROKE INTERVENTION FOR LARGE VESSEL OCCLUSION WITH COMBINED STENT RETRIEVER AND SUCTION THROMBECTOMY (SOLUMBRA TECHNIQUE): A RETROSPECTIVE ANALYSIS OF 85 PATIENTS}

${ }^{1} \mathrm{~J}$ Wong, ${ }^{2} \mathrm{~N}$ Telischak, ${ }^{2} \mathrm{~J}$ Heit, ${ }^{1} \mathrm{~A}$ Moraff, ${ }^{1} \mathrm{R}$ Dodd, ${ }^{2} \mathrm{H}$ Do, ${ }^{2} \mathrm{M}$ Marks. ${ }^{1}$ Neurosurgery, Stanford, Stanford, $C A ;{ }^{2}$ Radiology, Stanford, Stanford, CA

\subsection{6/neurintsurg-2016-012589.155}

Introduction The superiority of mechanical thrombectomy over medical management has been established in recent randomized controlled trials, however, controversy remains over the most effective and safest technique for clot retrieval. Mechanical thrombectomy for large vessel occlusion (LVO) can be performed through aspiration alone with the ADAPT technique, or combined stent retriever with aspiration either in the cervical vasculature using a proximal balloon guide, or directly at the clot face with an intermediate catheter, the so-called "Solumbra" technique. The purpose of this study was to evaluate the efficacy and safety of the Solumbra technique at our institution.

Methods A retrospective chart review was conducted of all patients undergoing endovascular stroke treatment for LVO using the Solumbra technique between January 2014 and March 2016. The Solumbra technique consisted of deployment of a stent retriever (Solitaire, Trevo or Mindframe) distal to the clot with an intermediate catheter (5 Max Ace, Ace 64 or SOFIA) at the clot face. Patient demographic data, stroke presentation, treatment details, and complications were recorded. The primary outcome was successful TICI 2 B/3 reperfusion and the number of passes for revascularization. Secondary outcome measures were complication rates, including symptomatic intracranial hemorrhage ( $\mathrm{PH} 2$ hemorrhage with a NIHSS increase $>4$ ) and mRS $\leq 2$ on discharge. Embolization into new territory was based on new post-procedure MRI DWI lesions.

Results The Solumbra technique was performed for LVO in 85 patients (37 male, 48 female) with a mean age of 71.4 years (range 28-93). The mean NIHSS on presentation was 14 (IQR 10-19), and IV tPA was administered in 65\% of patients. Vessel occlusion was located predominantly in the anterior circulation in the ICA terminus ( $\mathrm{n}=12,14 \%)$, M1 ( $\mathrm{n}=51,60 \%)$ and M2 ( $\mathrm{n}=16,19 \%)$ segments, with Basilar occlusion occurring in 7 patients (8\%). Successful reperfusion (TICI $2 \mathrm{~B} / 3$ ) was achieved in $88 \%$ of patients, consisting of TICI 3 in 38 patients (45\%). The mean number of passes for revascularization was 1.9 (SD 1.3) and time from access to revascularization was 52.7 minutes (95\% CI 45.3-60.1). Mean NIHSS on discharge was 7 (IQR 2-12) with good functional outcome $(\mathrm{mRS} \leq 2)$ present in $44 \%$ of patients, and mortality $(\mathrm{mRS}=6)$ rate of $12 \%$. Complications included parenchymal hemorrhagic transformation (18\%), symptomatic intracranial hemorrhage $(4.7 \%)$ and emboli in new territory based on new MRI lesions (7\%).

Conclusion The Solumbra technique compares favourably to other thrombectomy techniques used for acute stroke intervention. It achieved a TICI $2 \mathrm{~B} / 3$ reperfusion in $88 \%$ of patients, with an average number of 1.9 passes. Good functional outcome was achieved in $44 \%$ of patients on discharge, with a symptomatic intracranial hemorrhage rate of $4.7 \%$.

Disclosures J. Wong: None. N. Telischak: None. J. Heit: None. A. Moraff: None. R. Dodd: None. H. Do: None. M. Marks: None.

\section{E-084 LONG-TERM CLINICAL OUTCOMES IN PATIENTS WITH ACUTE ISCHEMIC STROKES TREATED WITH MECHANICAL THROMBECTOMY WHO HAD MODERATE OR MODERATE SEVERE DISABILITY AT 90 DAYS}

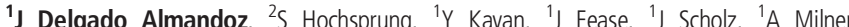
${ }^{2} \mathrm{P}$ Roohani, ${ }^{3} \mathrm{M}$ Mulder, ${ }^{2} \mathrm{~T}$ Hehr, ${ }^{2} \mathrm{R}$ Tarrel, ${ }^{4} \mathrm{D}$ Chappuis, ${ }^{2} \mathrm{M}$ Young. ${ }^{1}$ Neurointerventional Radiology, Abbott Northwestern Hospital, Minneapolis, MN; ${ }^{2}$ Vascular Neurology, Abbott Northwestern Hospital, Minneapolis, MN; ${ }^{3}$ Critical Care Medicine, Abbott Northwestern Hospital, Minneapolis, MN; ${ }^{4}$ Courage Kenny Rehabilitation Institute, Abbott Northwestern Hospital, Minneapolis, MN

\subsection{6/neurintsurg-2016-012589.156}

Background and purpose Long-term clinical outcomes in patients with acute ischemic stroke (AIS) treated with mechanical thrombectomy (MT) are not well understood. This study aims to determine long-term clinical outcomes in a consecutive cohort of patients with AIS treated with MT who had moderate or moderate severe disability at 90 day follow-up.

Methods Our prospectively-maintained institutional neurointerventional database was queried to identify all patients with AIS treated with MT at our institution from July 1st, 2011 until September 30th, 2015 who had moderate (modified Rankin Scale, mRS, 3) or moderate severe (mRS 4) disability at the time of the 90 day follow-up. At least 6 months after stroke onset, medical records were reviewed and/or phone interviews were conducted by a nurse certified in the mRS and independent of the MT procedure to determine the mRS at the time of last clinical contact. Baseline clinical and radiological characteristics were also obtained.

Results Forty patients met the study's inclusion criteria, comprising 29\% of patients treated with MT at our institution during the study period. Twenty patients were male $(50 \%)$ and 20 female $(50 \%)$. At presentation, 14 patients had a history of atrial fibrillation (35\%), 28 hypertension (70\%) and 13 diabetes mellitus (33\%). Mean age was 69.8 years. Mean admission NIHSS was 18.2. Twenty-five patients received intravenous tissue plasminogen activator (63\%). Thrombus location was M1 segment in 18 patients (45\%), internal carotid artery terminus in 14 patients $(35 \%)$, basilar artery in 4 patients (10\%) and M2/M3 segment in 4 patients (10\%). Discharge disposition was acute inpatient rehabilitation in 26 patients $(65 \%)$ and skilled nursing facility in 14 patients (35\%). At the time of the 90 day follow-up visit, 18 patients had an mRS of $3(45 \%)$ and 22 had an mRS of $4(55 \%)$. All 
patients continued to undergo outpatient rehabilitation treatment after the 90 day follow-up visit. Mean time to longterm clinical follow-up was 21.5 months after stroke onset (median 18.8 months, range 6-49 months). The table illustrates the clinical outcomes at the time of long-term followup. Between the 90 day and long-term follow-ups, 12 patients (30\%) had a reduction of at least 1 point in the mRS, whereas 10 patients $(25 \%)$ had an increase of at least 1 point in the mRS. At the time of long-term follow-up, 23 patients lived at home (58\%), 3 in an assisted living facility (8\%), 6 in a skilled nursing facility (15\%) and 8 were deceased (20\%). Four patients had experienced a recurrent AIS (10\%) by the time of long-term follow-up.

Conclusion Among patients with AIS treated with MT who had moderate or moderate severe disability at 90 days, a considerable minority $(30 \%)$ demonstrated a reduction in longterm disability with continued outpatient rehabilitation.

\begin{tabular}{|c|c|c|c|c|c|}
\hline \multicolumn{6}{|c|}{$\begin{array}{l}\text { Long-term outcomes in Pts w moderate or moderate severe disability after } \\
\text { mechanical thrombectomy }\end{array}$} \\
\hline & \multicolumn{5}{|c|}{ Long-term modified rankin scale } \\
\hline & 2 & 3 & 4 & 5 & 6 \\
\hline \multirow[t]{2}{*}{ All Patients $(n=40)$} & 5 & 18 & 7 (17.5\%) & 2 & 8 \\
\hline & $(12.5 \%)$ & $(45 \%)$ & & $(5 \%)$ & $(20 \%)$ \\
\hline Patients with Moderate Disability & 4 & 11 & 0 & 0 & 3 \\
\hline at 90 Days (mRS $3, \mathrm{n}=18$ ) & $(22.2 \%)^{*}$ & $(61.1 \%)$ & & & $(16.7 \%)$ \\
\hline Patients with Moderate Severe & 1 & 7 & 7 & 2 & 5 \\
\hline Disability at 90 Days & $(4.5 \%)^{*}$ & $(31.8 \%)^{*}$ & $(31.8 \%)$ & $(9.1 \%)$ & $(22.7 \%)$ \\
\hline$(m R S 4, n=22)$ & & & & & \\
\hline
\end{tabular}

Disclosures J. Delgado Almandoz: 2; C; Medtronic Neurovascular, Penumbra, Inc. S. Hochsprung: None. Y. Kayan: 2; C; Medtronic Neurovascular, Penumbra, Inc. J. Fease: None. J. Scholz: None. A. Milner: None. P. Roohani: None. M. Mulder: None. T. Hehr: None. R. Tarrel: None. D. Chappuis: None. M. Young: None.

\section{E-085 GOOD BASELINE ANGIOGRAPHIC COLLATERAL FLOW CORRELATES WITH SUCCESSFUL RECANALIZATION AND CLINICAL OUTCOME AFTER ENDOVASCULAR TREATMENT FOR ACUTE ISCHEMIC STROKE WITHIN 24 HOURS FROM ONSET}

1J Seo, ${ }^{1} \mathrm{E} \mathrm{Kim},{ }^{2} \mathrm{H}$ Jeong. ${ }^{1}$ Neurology, Inje University colleage of Medicine, Busan, Republic of Korea; ${ }^{2}$ Radiology, Inje University colleage of Medicine, Busan, Republic of Korea

\subsection{6/neurintsurg-2016-012589.157}

Background Recanalization status is the strongest predictor of clinical outcome in patients undergoing endovascular treatment (EVT). Although the time to reperfusion remains crucial even with EVT, the previous meta-analysis demonstrated that recanalization up to 24 hours after acute ischemic stroke (AIS) onset is strongly associated with improved functional outcomes and reduced mortality. We evaluated the relationships among angiographic collateral flow, successful recanalization, and clinical outcome after EVT in patients experiencing AIS within 24 hours of onset.
Methods We assessed patients were experiencing acute anterior circulation ischemic stroke who underwent EVT between 2011 and 2015. Patients with large artery occlusion of anterior circulation and clinical diagnosis of AIS, within 24 hours of first found abnormal time (FAT), were included in the study.

Results One hundred seventy-three patients met the inclusion criteria. Mean age was $68.3 \pm 12.6$ years, and median National Institutes of Health Stroke Scale score was 14 (range, 5-29). Median time from FAT to arrival was 92 minutes. Overall successful recanalization, defined by the Modified Thrombolysis In Cerebral Infarction scale grade 2 b-3, was achieved in $65.3 \%$ of patients, and good clinical outcome, as defined by the modified Rankin Scale (mRS 0-2), was achieved in $47.4 \%$ of patients. For successful $r$ the angiographic collateral grade was the independent factor. In multiple logistic regression analysis, the angiographic collateral grade was independently associated with clinical outcome after adjusting for other variables (odds ratio, 5.96; 95\% CI, 1.7620.19).

Conclusions Our data showed that angiographic collateral grade was a strong independent predictor of successful recanalization after EVT and good clinical outcome in AIS patients when applied up to 24 hours from FAT. Consequently, the good angiographic collateral flow may help predict successful recanalization and better clinical outcomes after EVT in patients with AIS.

Disclosures J. Seo: None. E. Kim: None. H. Jeong: None.

\section{E-086 MANDIBULAR AVM DIAGNOSIS AND CURATIVE TREATMENT}

W Yakes. Vascular Malformation Center, Englewood, CO

\subsection{6/neurintsurg-2016-012589.158}

Purpose To determine optimal management strategies for the treatment of mandibular AVM.

Materials and methods Twelve patients (9 females, 3 males), age 9-14; mean age 10, underwent endovascular therapy to treat their mandibular AVMs. Nine patients had distinct intraosseous AVMs. Three had multiple facial and intra-maxillary AVMs requiring treatment. Outside institutions recommended massive hemi-facial resections in these patients. Four patients had prior PVA and gel foam embolization, one patient had a lip graft, one had prior mandible surgery, all that had failed.

Results All twelve patients have demonstrated MR and angiographic cure of their AVMs. One patient's therapy is not completed and is on-going. The patients mandibular AVM is cured, a third AVM in this patient in the infratemporal fossa is still undergoing treatment. The follow-up range is 11 months - 41 months, with a mean follow-up of 29 months. No complications were noted in treatment of mandibular AVMS. One patient required a minor gingival surgery after treatment of an additional intramaxillary AVM.

Conclusions Endovascular approaches to manage mandibular AVM can be curative. The intraosseous variety is largely a fistula between artery and vein within the bone. All respond well to endovascular ethanol therapy alone. Surgery was not required in any patient. Surprisingly no complications were encountered in this patient series. Long-term cures are noted in this patient series with endovascular approaches alone. No massive surgical resections in any patient, even in patients with multiple AVMs of the soft tissues, mandible and maxilla, 